\title{
Wall Contrast Enhancement of Thrombosed Intracranial Aneurysms at 7T MRI
}

\author{
(D)T. Sato, (D) T. Matsushige, (D) B. Chen, (D). Gembruch, (DP. Dammann, DR. Jabbarli, (D) M. Forsting, (D) A. Junker, (D). Maderwald, \\ (D)H.H. Quick, (D) M.E. Ladd, (D). Sure, and DK.H. Wrede
}

\begin{abstract}
BACKGROUND AND PURPOSE: The pathophysiology of wall contrast enhancement in thrombosed intracranial aneurysms is incompletely understood. This in vivo study aimed to investigate wall microstructures with gadolinium-enhanced 7T MR imaging.
\end{abstract}

MATERIALS AND METHODS: Thirteen patients with 14 thrombosed intracranial aneurysms were evaluated using a 7T whole-body MR imaging system with nonenhanced and gadolinium-enhanced high-resolution MPRAGE. Tissue samples were available in 5 cases, and histopathologic findings were correlated with 7T MR imaging to identify the gadolinium-enhancing microstructures.

RESULTS: Partial or complete inner wall enhancement correlated with neovascularization of the inner wall layer and the adjacent thrombus. Additional partial or complete outer wall enhancement can be explained by formation of vasa vasorum in the outer aneurysm wall layer. The double-rim enhancement correlated with perifocal edema and wall histologic findings suggestive of instability.

CONCLUSIONS: Two distinct aneurysm wall microstructures responsible for gadolinium enhancement not depictable at lower spatial resolutions can be visualized in vivo using high-resolution gadolinium-enhanced 7T MR imaging.

$\mathbf{T}$ reatment of thrombosed intracranial aneurysms, most of which are large $(12-24 \mathrm{~mm})$ or giant $(>25 \mathrm{~mm})$, is complex and associated with a high risk of complications. ${ }^{1,2}$ Previous studies have suggested a different pathophysiology of thrombosed intracranial aneurysms compared with nonthrombosed aneurysms. ${ }^{3,4}$ The mechanisms on the cellular level remain unclear, but histopathologic studies have improved our understanding of the pathophysiology. ${ }^{5}$

Histopathologic studies regarding the thrombosed intracranial aneurysm wall showed neovascularization or vascular chan-

Received February 15, 2019; accepted after revision April 25.

From the Department of Neurosurgery (T.S., T.M., B.C., O.G., P.D., R.J., U.S., K.H.W.), University Hospital Essen, Department of Diagnostic and Interventional Radiology and Neuroradiology (M.F.), Institute of Neuropathology (A.J.), High Field and Hybrid MR Imaging (H.H.Q.), and Erwin L. Hahn Institute for Magnetic Resonance Imaging (T.S., T.M., B.C., O.G., P.D., S.M., H.H.Q., M.E.L., K.H.W.), University Duisburg-Essen, Essen, Germany; Department of Neurosurgery (T.S.), Fukushima Medical University, Fukushima, Japan; Department of Neurosurgery (T.M.), Graduate School of Biomedical and Health Sciences, Hiroshima University, Hiroshima, Japan; Department of Neurosurgery and Interventional Neuroradiology (T.M.), Hiroshima City Asa Citizens Hospital, Hiroshima, Japan; Medical Physics in Radiology (M.E.L.), German Cancer Research Center, Heidelberg, Germany; and Faculty of Physics and Astronomy and Faculty of Medicine (M.E.L.), University of Heidelberg, Heidelberg, Germany.

Karsten H. Wrede, Bixia Chen, and Oliver Gembruch were supported by the University Duisburg-Essen (Programm zur internen Forschungsförderung Essen).

Please address correspondence to Taku Sato, MD, PhD, University Hospital Essen, Department of Neurosurgery, Hufelandstr 55, 45147 Essen, Germany; e-mail:

Taku.Sato@uk-essen.de

http://dx.doi.org/10.3174/ajnr.A6084 nel lining of the intima, as well as thrombus formation in the aneurysm dome far away from the parent artery. These findings seem to play an important role in aneurysm growth. ${ }^{4,6-8}$ In rare cases of giant fusiform thrombosed aneurysms, persistent growth was observed even after proximal occlusion and trapping. ${ }^{3,9}$

Wall-enhancement patterns of thrombosed intracranial aneurysms on CT and MR imaging are recognized as one of the clinical-alert signs indicating their unstable behavior. ${ }^{3,9}$ However, in current imaging techniques, wall enhancement is visualized as a single rim due to insufficient spatial resolution, and the microstructures cannot be discriminated in vivo.

Recent research has shown that ultra-high spatial resolution 7T MR imaging is an excellent tool for in vivo visualization of aneurysm microstructures. ${ }^{10}$ This study therefore aimed to investigate the microstructure of thrombosed intracranial aneurysm wall-enhancement patterns using ultra-high-field $7 \mathrm{~T}$ contrastenhanced MR imaging with direct comparison with histopathologic findings.

\section{MATERIALS AND METHODS Study Design and Patient Cohort}

The study was conducted according to the principles of the Declaration of Helsinki and was Health Insurance Portability and Accountability Act-compliant. The authorized ethics committee of the University Duisburg-Essen approved the study, and all patients provided written informed consent before the examination. 


\begin{tabular}{|c|c|c|c|c|c|c|c|c|c|c|}
\hline $\begin{array}{c}\text { Aneurysm } \\
\text { No. }\end{array}$ & $\begin{array}{c}\text { Patient } \\
\text { No. }\end{array}$ & $\begin{array}{l}\text { Age } \\
\text { (yr) }\end{array}$ & Sex & Location & $\begin{array}{c}\text { Maximum } \\
\text { Diameter } \\
(\mathrm{mm})\end{array}$ & Thrombosis & $\begin{array}{c}\text { Wall } \\
\text { Contrast-Enhancement } \\
\text { Pattern }\end{array}$ & $\begin{array}{c}\text { Perifocal } \\
\text { Edema }\end{array}$ & Treatment & Pathology \\
\hline $1^{a}$ & 2 & 75 & $M$ & BA & 27.2 & Complete & Double (partial) & + & Observation & - \\
\hline $2^{a}$ & 3 & 80 & M & MCA & 29.0 & Complete & Double (complete) & + & Observation & - \\
\hline $3^{a}$ & 4 & 61 & $\mathrm{~F}$ & MCA & 26.0 & Partial & Double (complete) & + & Clipping & + \\
\hline 4 & 8 & 56 & M & MCA & 29.6 & Partial & Double (Partial) & + & Clipping & + \\
\hline 5 & 10 & 53 & M & MCA & 14.2 & Partial & Double (Partial) & + & Clipping & + \\
\hline 6 & 13 & 44 & $\mathrm{~F}$ & MCA & 28.2 & Partial & Double (Partial) & + & Clipping & + \\
\hline 7 & 5 & 55 & M & MCA & 25.0 & Partial & Double (complete) & + & Clipping & - \\
\hline 8 & 5 & 55 & M & $\mathrm{dACA}$ & 11.0 & Partial & Single & - & Clipping & - \\
\hline $9^{a}$ & 1 & 56 & $\mathrm{~F}$ & ICA & 37.9 & Partial & Single & - & Endovascular ${ }^{b}$ & - \\
\hline 10 & 6 & 52 & $\mathrm{~F}$ & ICA & 23.2 & Partial & Single & - & Endovascular & - \\
\hline 11 & 7 & 63 & $\mathrm{~F}$ & ICA & 21.4 & Partial & Single & - & Endovascular & - \\
\hline 12 & 9 & 70 & $\mathrm{~F}$ & MCA & 15.6 & Partial & Single & - & Clipping & - \\
\hline 13 & 11 & 43 & M & ICA & 36.0 & Partial & Single & - & Endovascular b & - \\
\hline 14 & 12 & 50 & $\mathrm{~F}$ & MCA & 21.6 & Partial & Single & - & Clipping & + \\
\hline
\end{tabular}

Note:-BA indicates basilar artery; dACA, distal anterior cerebral artery; +, Yes; -, No.

${ }^{a}$ Aneurysms 1, 2, 3 and 9 have been reported in the context of giant intracranial aneurysms imaged at 7T MRI. ${ }^{10}$

b Parent vessel occlusion.

Patients were prospectively included between January 2011 and November 2018. The study cohort comprised 6 men and 7 women with an average age of 58.3 years (range, 43-80 years). Inclusion criteria were the following: 1) patients with a thrombosed intracranial aneurysm diagnosed by digital subtraction angiography and conventional CT or 3T MR imaging, 2) 18 years of age or older, and 3) able to give informed consent. Exclusion criteria were the following: 1) presence of a cardiac pacemaker or any other electronic implant, 2) pregnancy or breastfeeding, 3) claustrophobia, or 4) patients needing permanent monitoring (eg, subarachnoid hemorrhage). Aneurysms 1, 2, 3, and 9 have previously been presented in a different context of giant intracranial aneurysm imaging. ${ }^{10}$

\section{High-Resolution 7T MR Imaging}

All subjects were evaluated with a 7T whole-body MR system (Magnetom 7T; Siemens, Erlangen, Germany) equipped with a 1/32-channel $\mathrm{Tx} / \mathrm{Rx}$ head radiofrequency coil (Nova Medical, Wilmington, Massachusetts). The gradient system provides 40$\mathrm{mT} / \mathrm{m}$ maximum amplitude and a slew rate of $200 \mathrm{mT} / \mathrm{m} / \mathrm{ms}$. A modified MPRAGE sequence was obtained with the following parameters: $\mathrm{FOV}=270 \times 236 \mathrm{~mm}^{2}$, matrix $=384 \times 336$, resolution $=0.7 \times 0.7 \mathrm{~mm}^{2}$, slice thickness $=0.7 \mathrm{~mm}, \mathrm{TR}=2500 \mathrm{~ms}$, $\mathrm{TE}=1.54 \mathrm{~ms}$, flip angle $=7^{\circ}$, bandwidth $=570 \mathrm{~Hz} /$ pixel, acquisition time $=6$ minutes 13 seconds. ${ }^{11,12}$ Gadolinium contrastenhanced images were acquired 10 minutes after intravenous administration of a gadobutrol-based macrocyclic contrast agent ( $1 \mathrm{mmol} / \mathrm{mL} / 10 \mathrm{~kg}$ of body weight). High-resolution T2-weighted images were acquired by a modified TSE sequence with the following parameters: $\mathrm{FOV}=176 \times 256 \mathrm{~mm}^{2}$, matrix $=512 \times 384$, resolution $=0.45 \times 0.5 \mathrm{~mm}^{2}$, slice thickness $=2 \mathrm{~mm}, \mathrm{TR}=6000 \mathrm{~ms}, \mathrm{TE}=100 \mathrm{~ms}$, flip angle $=180^{\circ}$, bandwidth $=264 \mathrm{~Hz} /$ pixel, acquisition time $=4$ minutes 1 second.

\section{Image Analysis}

Two raters assessed the following characteristics in multiplanar image reconstruction using an open-source medical image viewer (Horos, Version 2.0.1; http://www.horosproject.org/) in consen- sus reading: 1) aneurysm wall contrast patterns and 2) presence of perifocal edema.

\section{Histopathologic Examination}

Histopathologic samples of 5 thrombosed intracranial aneurysms (aneurysms 3, 4, 5, 6, and 14), harvested during microsurgical clipping of the aneurysm, were analyzed and compared with MR imaging findings. Histopathologic sections from areas corresponding to MR imaging ROIs were prepared with $5-\mu \mathrm{m}$ thickness and were stained with hematoxylin-eosin, Verhoeff-Van Gieson, Prussian blue, and CD68. The degree of macrophage infiltration was evaluated with the ImageJ software package (National Institutes of Health, Bethesda, Maryland). ${ }^{13}$ First, CD68 immunostainings were digitized using an Aperio AT2 slide scanner (Leica Microsystems, Wetzlar, Germany). From the scanned files, 4 images with an edge length of $300 \mu \mathrm{m}$ and the highest macrophage activity covering the complete thickness of the aneurysm wall were extracted for further analysis. The CD68-positive areas of all 4 ROIs were measured and calculated as a percentage using the ImageJ functions "color threshold" and "analyze particles."

\section{Statistical Analysis}

Due to the limited number of cases, only small-sample tests could be applied. A $\chi^{2}$ test was used to evaluate the perifocal edema and the contrast-enhancement pattern. Correlation between size and contrast-enhancement patterns was analyzed by a MannWhitney $U$ test. Significance level $\alpha$ was defined as $P<.05$.

\section{RESULTS}

All patients were examined without any adverse events, and all MR imaging sequences were successfully acquired. Basic demographic data for all patients and major anatomic features of the aneurysms are summarized in the Table. Imaging studies revealed complete thrombosis of 2 aneurysms and partial thrombosis in 12 aneurysms. One patient (patient 5) had 2 aneurysms (both with partial thrombosis). The mean diameter of aneurysms was 24.7 $\mathrm{mm}$ (standard deviation, $7.4 \mathrm{~mm}$; range, $11.0-37.9 \mathrm{~mm}$ ). All aneurysms were partially or completely surrounded by brain parenchyma, with perifocal edema present in 7 aneurysms. All throm- 

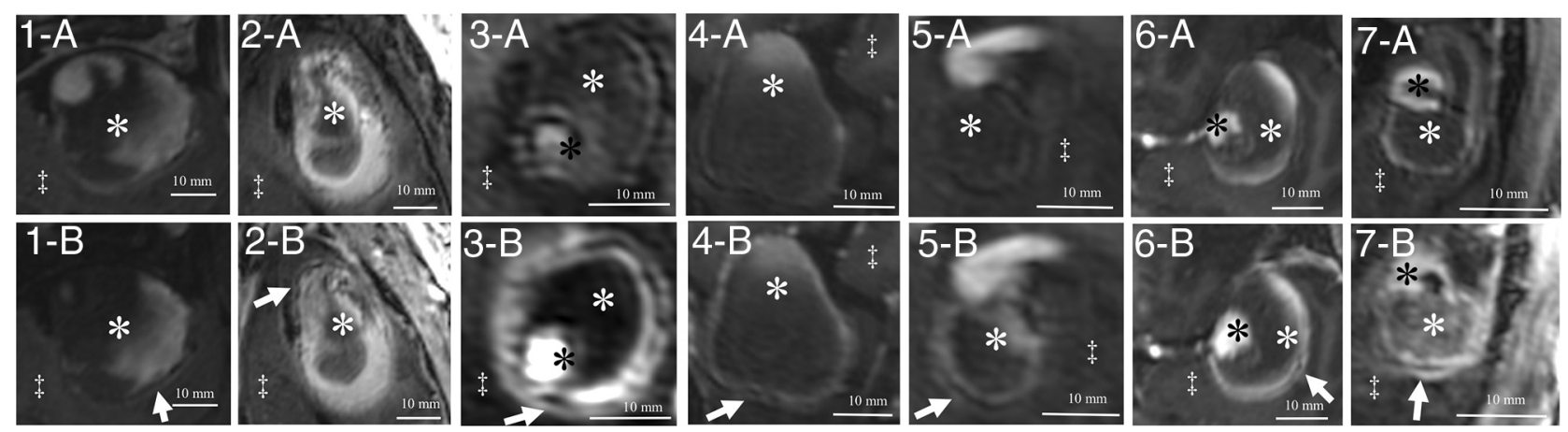

FIG 1. Delineation of thrombosed intracranial aneurysms ( $A$, Nonenhanced MPRAGE; $B$, Gadolinium-enhanced MPRAGE) with double-rim (aneurysms 1-7) enhancement patterns. Identical structures are marked in all subfigures as follows: White arrows indicate a double-rim pattern of the aneurysm walls; white asterisks, intraluminal thrombus; black asterisks, aneurysm lumen; double daggers, brain parenchyma.
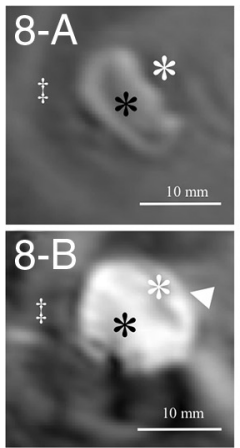
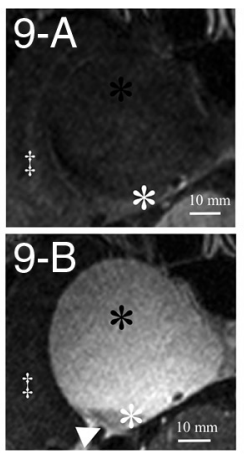
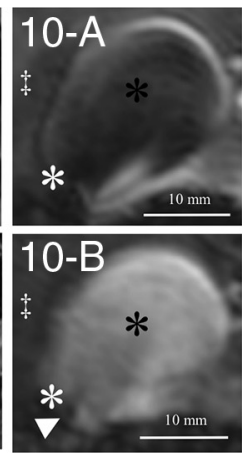
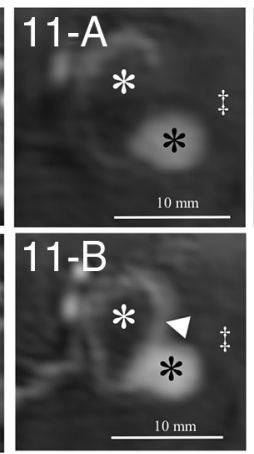
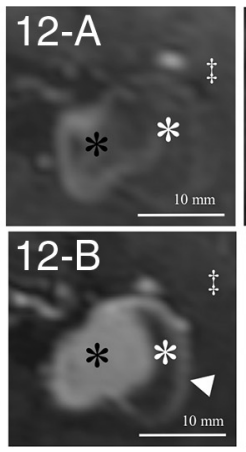
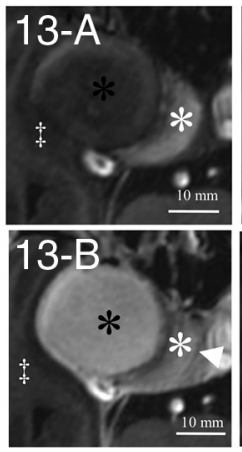
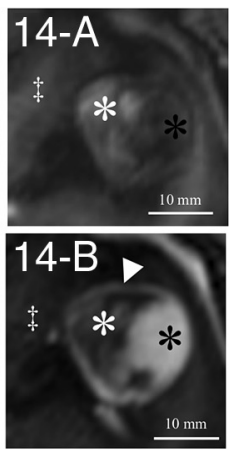

FIG 2. Delineation of thrombosed intracranial aneurysms ( $A$, Nonenhanced MPRAGE; $B$, Gadolinium-enhanced MPRAGE) with single-rim (aneurysms 8-14) enhancement patterns. Identical structures are marked in all subfigures as follows: White asterisks indicate intraluminal thrombus; black asterisks, aneurysm lumen; double daggers, brain parenchyma; white arrowheads, single-rim pattern of the aneurysm walls.

bosed intracranial aneurysms showed partial or complete rim enhancement of the inner wall layer (Figs 1 and 2). Additionally, in 7 aneurysms, the outer aneurysm wall layer showed either partial or complete rim enhancement (Fig 1). All thrombosed intracranial aneurysms with double-rim enhancement presented with perifocal edema $(P<.01)$. The maximum aneurysm diameter and presence of perifocal edema showed no correlation $(P=$ .456).

In 5 aneurysms, partial resection of the aneurysm dome was required during the microsurgical procedure to expose the neck, and these specimens were suitable for histologic examination. Four of these aneurysms presented with perifocal edema and double-rim enhancement (Fig 3). The thick wall showed vascularization of the intima and the fresh thrombus adjacent to the inner aneurysm layer. The outer signal of the double-rim enhancement correlated with vasa vasorum developing in the outer aneurysm wall layer and macrophage invasion in the media and adventitia. The region with only single-rim enhancement showed an undeveloped vasa vasorum and fewer macrophages (Fig 4). The average CD68-positive areas in the 4 double-rim pattern aneurysm walls were large $(19.5 \% \pm 6.4 \%)$ compared with the single-rim case with a CD68-positive area $(13.9 \% \pm 7.5 \%)$.

\section{DISCUSSION}

The overall prevalence of thrombosed intracranial aneurysms is unknown, but postmortem series reported it to be $9 \%$ of all intracranial aneurysms. ${ }^{14}$ Nevertheless, large and giant aneurysms show partial thrombosis much more frequently $(48 \%-76 \%)$, and the frequency seems to correlate with aneurysm size. ${ }^{3,15}$ Furthermore, the presence of partial thrombosis has been identified as a clinical biomarker for aneurysm histologic findings suggestive of instability and risk of rupture. Both completely thrombosed aneurysms were treated conservatively, though aneurysm size, shape, and contrast-enhancement patterns indicated treatment. These patients ( 80 and 75 years of age) had severe comorbidities and therefore refused microsurgical treatment. Clinical impact of the presented findings remains unclear because the treatment indication was given in all presented cases due to large aneurysm sizes. Because the results cannot be generalized to smaller aneurysms, further studies in larger patient cohorts are needed.

Ollikainen et $\mathrm{al}^{16}$ have shown that aneurysm wall remodeling and histologic findings suggestive of instability are associated with chronic inflammation in histopathology. Lack of internal elastic lamina, erosion of the luminal endothelium, infiltration of inflammatory cells, apoptosis of smooth-muscle cells, and the presence of myointimal hyperplasia, fibrosis, and thrombus are characteristics of aneurysm wall remodeling. These processes are also associated with neovascularization of the intima and formation of vasa vasorum and finally wall degeneration. Neovascularization of the intima and within the thrombus as seen in the histopathology of thrombosed intracranial aneurysm walls farthest away from the parent artery has been reported previously and seems to play an important role in aneurysm growth, histologic findings suggestive of instability, and rupture. ${ }^{4,17}$

Frösen et $\mathrm{al}^{6}$ have also proposed several explanations for iron 

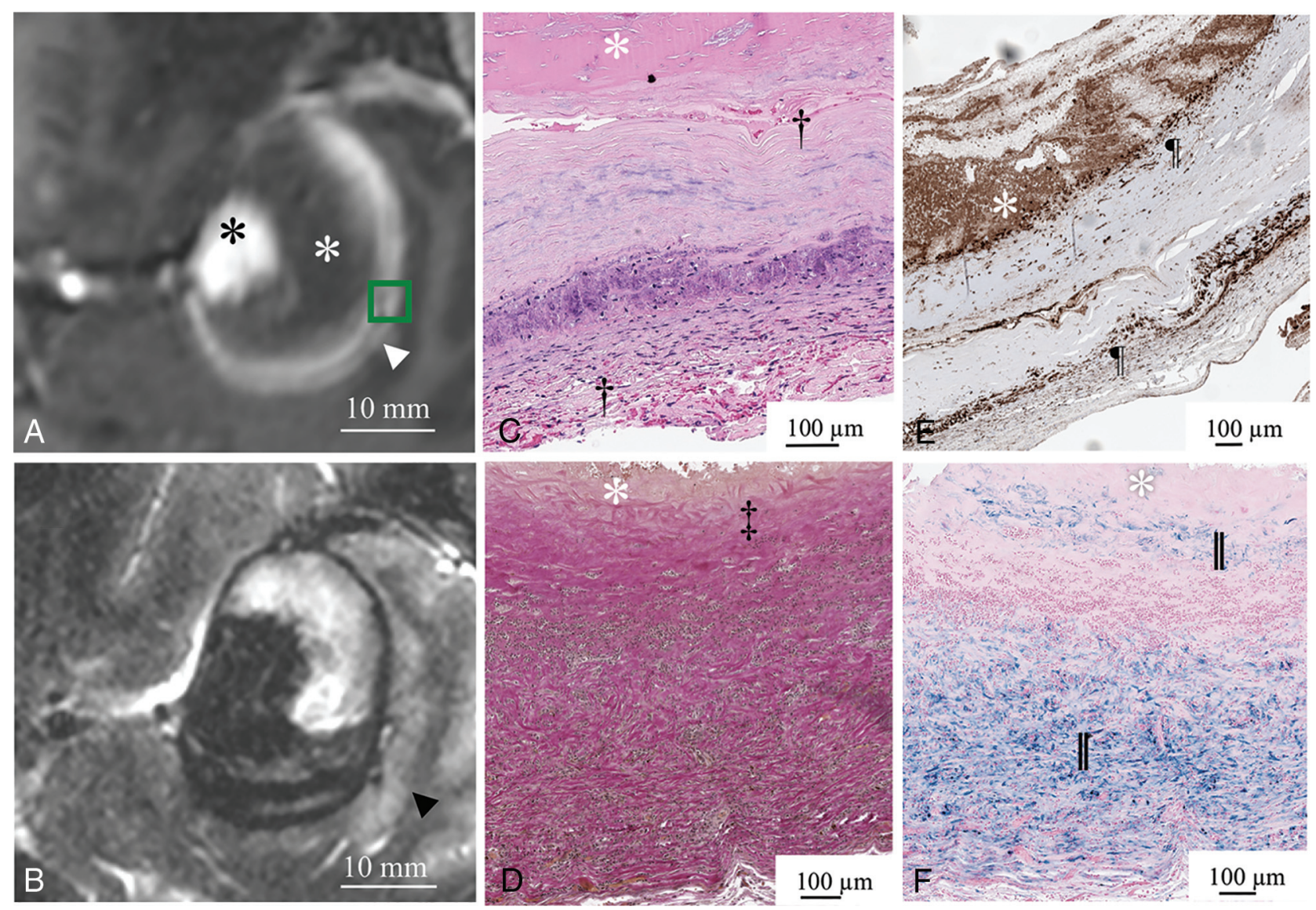

FIG 3. Gadolinium-enhanced MPRAGE shows a double-rim pattern of the aneurysm wall (white arrowhead) in aneurysm 6 (white asterisk, intraluminal thrombus; black asterisk, aneurysm lumen). The green box indicates the area of the histopathologic specimen (A). Perifocal edema (black arrowhead) adjacent to the double-rim enhancement delineated in T2-weighted MR imaging (B). The white asterisk indicates intraluminal thrombus $(C-F)$. Good correspondence between the double-rim pattern of the aneurysm wall and neovascularization of the inner wall layer (upper dagger) and developed vasa vasorum (lower dagger) in hematoxylin-eosin staining (C). Loss of elastic fibers in the inner aneurysm wall layer in Van Gieson elastic staining (double daggers) (D). Abundant macrophages (pilcrow sign) in the inner and outer layers of the aneurysm wall visualized in CD68 immunostaining (E). Iron deposition (double vertical lines) in the inner and outer aneurysm wall layers stained with Prussian blue $(F)$.

deposition and macrophage accumulation in unstable aneurysm walls. Interstitial iron results from erythrocytes seeping into the interstitial space from leaky vessels. This iron is then phagocytosed by macrophages migrating into the aneurysm wall. Prussian blue staining and immunostaining (eg, CD163) can reveal these processes in histologic specimens. On the other hand, macrophage infiltration was also present in most aneurysm walls without iron deposition, supporting the assumption that different inflammatory processes are involved in macrophage migration. The average CD68-positive areas in the 4 double-rim-pattern aneurysm walls were large $(19.5 \% \pm 6.4 \%)$ compared with the singlerim case with a CD68-positive area $(13.9 \% \pm 7.5 \%)$, but there were too few histopathologic samples to statistically evaluate the difference. Nevertheless, the macrophage infiltration seems to be one of the important pathophysiologic mechanisms involved in wall enhancement in thrombosed aneurysms. Even though part of thrombosed intracranial aneurysm pathophysiology has already been explained, several aspects still remain unclear, and in vivo evaluation is not feasible with current clinical imaging methods. ${ }^{3,9,15,18}$

Vessel wall imaging with gadolinium-enhanced MR imaging has become more popular in the past years and might be a clinical biomarker for histologic findings suggestive of aneurysm instability. ${ }^{19,20}$ Nevertheless, due to the variety of different vessel wall imaging techniques, generalizability of the published results remains limited. Restricted by spatial resolution, wall enhancement has been described as a partial or complete single rim using current clinical CT or MR imaging systems, and the actually enhancing wall microstructure could not be identified in vivo. MR imaging at $7 \mathrm{~T}$ has demonstrated a great advantage in detecting aneurysm wall microstructures. The higher signal-to-noise ratio makes voxel sizes feasible for in vivo imaging that are significantly smaller than the wall thickness. ${ }^{10-12}$ The presented study reports distinct single- and double-rim enhancement patterns in thrombosed intracranial aneurysms using ultra-high-field 7T MR imaging for the first time. Quantifying a normalized degree of enhancement was not feasible, due to intersubject variance of pharmacokinetics and non-normalized MR imaging signal intensities. Quantitative T1 and T2 mapping sequences might make this possible in the future.

Our results suggest that 2 distinct aneurysm wall microstructures are responsible for gadolinium wall enhancement in thrombosed intracranial aneurysms that cannot be discriminated at a lower spatial resolution. The partial or complete inner wall en- 

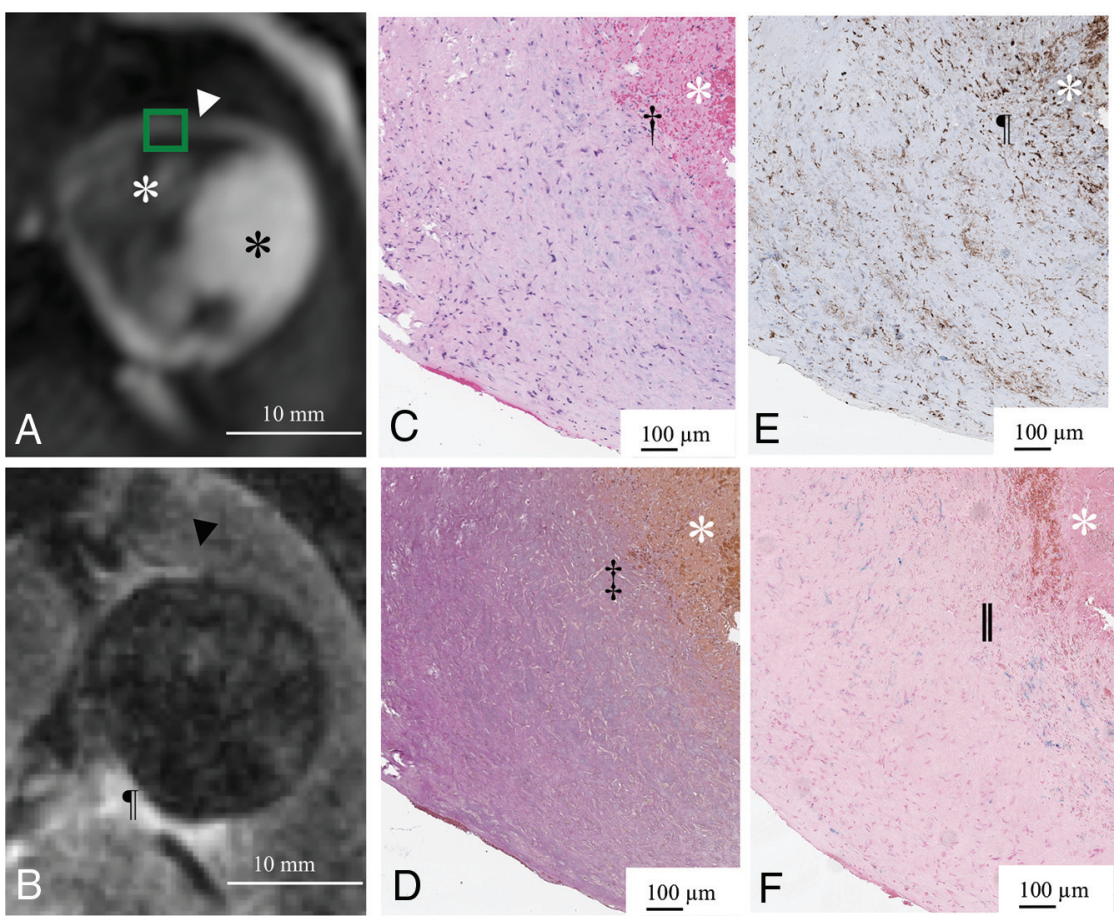

FIG 4. Gadolinium-enhanced MPRAGE shows a single-rim pattern (white arrowhead) in aneurysm 14 (white asterisk, intraluminal thrombus; black asterisk, aneurysm lumen). The green box indicates the area of the histopathologic specimen (A). No perifocal edema (black arrowhead) adjacent to the single-rim enhancement can be delineated in T2-weighted MR imaging. Cisternal CSF is delineated with hyperintense signal (pilcrow sign) (B). The white asterisk indicates intraluminal thrombus $(C-F)$. Good correspondence between the single-rim pattern of the aneurysm wall and neovascularization of the inner wall layer (dagger) and missing vasa vasorum of the outer wall layer in hematoxylin-eosin staining (C). Loss of elastic fibers in the inner aneurysm wall layer in Van Gieson elastic staining (double daggers) (D). Abundant macrophages (pilcrow sign) in the inner layer and few in the outer layer of the aneurysm wall visualized in CD68 immunostaining (E). Iron deposition (double vertical lines) in the inner and less in the outer aneurysm wall layers stained with Prussian blue $(F)$.

hancement seen in all presented cases correlates with neovascularization of the inner wall layer and the adjacent thrombus. Furthermore, neovascularization of the inner wall layer has also been reported in histologic examinations of nonthrombosed aneurysms. ${ }^{8}$ The additional partial or complete outer wall enhancement seen in 7 of the presented patients (Table) can be explained by formation of vasa vasorum in the outer aneurysm wall layer. This double-rim pattern correlates with wall degeneration and histologic findings suggestive of instability. Furthermore, the vasa vasorum interface with pial vessels and therefore induce perifocal edema. These results are in line with previously proposed perifocal edema induction, not by mass effect but by direct interaction of the aneurysm wall with the pial surface. ${ }^{15}$ If direct correlation between perifocal edema adjacent to the aneurysm wall and the absence or presence of double-rim wall enhancement in 7T MR imaging can be shown in a larger patient cohort, these results would have an important clinical impact because perifocal edema can be easily visualized using lower magnetic-field-strength MR imaging. On the other hand, the pathophysiology of double-rim wall enhancement and perifocal edema is still not completely understood. Cavernous ICA aneurysms were not associated with perifocal edema, even though they exert mass effect on the brain, and their separation from the brain parenchyma by the dura mater may prevent the diffusion of edema-inducing factors despite the presence of partial thrombosis. Dengler et $\mathrm{al}^{15}$ reported that direct contact between the partially thrombosed surface of an aneurysm and the brain parenchyma may be crucial for perifocal edema formation. However, histopathologic correlations were pending.

\section{Limitations}

There are some limitations to the current study. The study cohort comprised only 13 patients with 14 thrombosed intracranial aneurysms. This is mainly due to the low prevalence of thrombosed intracranial aneurysms and the even lower rate of unruptured thrombosed intracranial aneurysms. Histopathologic correlations were only possible in 5 aneurysms. Only 1 case with single-rim wall enhancement was available for histopathologic analysis, restricting the comparison of both enhancement patterns. Identical enhancement patterns could be shown in various locations, thus indicating that these patterns are a general feature of thrombosed intracranial aneurysms.

\section{CONCLUSIONS}

Two distinct aneurysm wall microstructures responsible for gadolinium enhancement not depictable at a lower spatial resolution can be visualized in vivo using high-resolution gadoliniumenhanced 7T MR imaging. Partial or complete inner wall enhancement correlates with neovascularization of the inner wall layer as well as the adjacent thrombus. Additional partial or complete outer wall enhancement can be explained by formation of vasa vasorum in the outer aneurysm wall layer. The double-rim enhancement correlates with perifocal edema and wall histologic findings suggestive of instability.

Disclosures: Bixia Chen—RELATED: Grant: Programm zur internen Forschungsförderung Essen grant, Comments: scientific grant of the University of Duisburg-Essen.* Oliver Gembruch—RELATED: Grant: Interne Forschungsförderung Essen, Comments: The grant is a scientific grant given by the University Duisburg-Essen. * Harald H. Quick—RELATED: Other: Siemens Healthcare, Erlangen, Germany, Comments: The Erwin L. Hahn Institute for MR Imaging, University Duisburg-Essen, has an established research cooperation with Siemens Healthcare, Erlangen, Germany. ${ }^{*}$ Karsten H. Wrede-RELATED: Grant: Programm zur internen Forschungsförderung Essen grant, Comments: grant for scientific work from the University Duisburg Essen.* *Money paid to the institution.

\section{REFERENCES}

1. Roccatagliata L, Guédin P, Condette-Auliac S, et al. Partially thrombosed intracranial aneurysms: symptoms, evolution, and therapeutic management. Acta Neurochir (Wien) 2010;152: 2133-42 CrossRef Medline

2. Miyamoto S, Funaki T, Iihara K, et al. Successful obliteration and shrinkage of giant partially thrombosed basilar artery aneurysms 
through a tailored flow reduction strategy with bypass surgery. J Neurosurg 2011;114:1028-36 CrossRef Medline

3. Krings T, Alvarez H, Reinacher P, et al. Growth and rupture mechanism of partially thrombosed aneurysms. Interv Neuroradiol 2007; 13:117-26 CrossRef Medline

4. Nagahiro S, Takada A, Goto S, et al. Thrombosed growing giant aneurysms of the vertebral artery: growth mechanism and management. J Neurosurg 1995;82:796-801 CrossRef Medline

5. Lehman VT, Brinjikji W, Mossa-Basha M, et al. Conventional and high-resolution vessel wall MRI of intracranial aneurysms: current concepts and new horizons. J Neurosurg 2018;128:969-81 CrossRef Medline

6. Frösen J, Tulamo R, Paetau A, et al. Saccular intracranial aneurysm: pathology and mechanisms. Acta Neuropathol 2012;123:773-86 CrossRef Medline

7. Cebral J, Ollikainen E, Chung BJ, et al. Flow conditions in the intracranial aneurysm lumen are associated with inflammation and degenerative changes of the aneurysm wall. AJNR Am J Neuroradiol 2017;38:119-26 CrossRef Medline

8. Tulamo R, Frösen J, Hernesniemi J, et al. Inflammatory changes in the aneurysm wall: a review. J Neurointerv Surg 2010;2:120-30 CrossRef Medline

9. Iihara K, Murao K, Yamada N, et al. Growth potential and response to multimodality treatment of partially thrombosed large or giant aneurysms in the posterior circulation. Neurosurgery 2008;63:83242; discussion 842-43 CrossRef Medline

10. Matsushige T, Chen B, Ringelstein A, et al. Giant intracranial aneurysms at 7T MRI. AJNR Am J Neuroradiol 2016;37:636-41 CrossRef Medline

11. Umutlu L, Theysohn N, Maderwald S, et al. 7 Tesla MPRAGE imaging of the intracranial arterial vasculature: nonenhanced versus contrast-enhanced. Acad Radiol 2013;20:628-34 CrossRef Medline
12. Wrede KH, Johst $\mathrm{S}$, Dammann $\mathrm{P}$, et al. Caudal image contrast inversion in MPRAGE at 7 Tesla: problem and solution. Acad Radiol 2012;19:172-78 CrossRef Medline

13. Schneider CA, Rasband WS, Eliceiri KW. NIH image to ImageJ: 25 years of image analysis. Nat Methods 2012;9:671-75 CrossRef Medline

14. Housepian EM, Pool JL. A systematic analysis of intracranial aneurysms from the autopsy file of the Presbyterian Hospital, 1914 to 1956. J Neuropathol Exp Neurol 1958;17:409-23 CrossRef Medline

15. Dengler J, Maldaner N, Bijlenga P, et al; Giant Intracranial Aneurysm Study Group. Perianeurysmal edema in giant intracranial aneurysms in relation to aneurysm location, size, and partial thrombosis. J Neurosurg 2015;123:446-52 CrossRef Medline

16. Ollikainen E, Tulamo R, Frösen J, et al. Mast cells, neovascularization, and microhemorrhages are associated with saccular intracranial artery aneurysm wall remodeling. $J$ Neuropathol Exp Neurol 2014;73:855-64 CrossRef Medline

17. Nakatomi H, Segawa H, Kurata A, et al. Clinicopathological study of intracranial fusiform and dolichoectatic aneurysms: insight on the mechanism of growth. Stroke 2000;31:896-900 CrossRef Medline

18. Schubiger O, Valavanis A, Wichmann W. Growth-mechanism of giant intracranial aneurysms; demonstration by CT and MR imaging. Neuroradiology 1987;29:266-71 CrossRef Medline

19. Matouk CC, Mandell DM, Günel M, et al. Vessel wall magnetic resonance imaging identifies the site of rupture in patients with multiple intracranial aneurysms: proof of principle. Neurosurgery 2013; 72:492-96; discussion 496 CrossRef Medline

20. Shimonaga $\mathrm{K}$, Matsushige $\mathrm{T}$, Ishii $\mathrm{D}$, et al. Clinicopathological insights from vessel wall imaging of unruptured intracranial aneurysms. Stroke 2018;49:2516-19 CrossRef Medline 